\title{
X-ray microfocus Computed Tomography: A powerful tool for structural and functional characterisation of 3D printed dosage forms
}

\author{
Christos I. Gioumouxouzis ${ }^{1}$, Orestis L. Katsamenis ${ }^{2 *}$, Dimitrios G. \\ Fatouros $^{1}$
}

\author{
${ }^{1}$ School of Pharmacy, Aristotle University of Thessaloniki, Department of \\ Pharmaceutical Technology, GR-54124 Thessaloniki, Greece \\ ${ }^{2} \mu$-VIS X-ray Imaging Centre, Faculty of Engineering and Physical Sciences, University \\ of Southampton, SO17 1BJ, Southampton, UK
}

Corresponding author: Dr. Orestis L. Katsamenis

$\mu$-VIS X-ray Imaging Centre

Building 5 / Room 1015 - Mailpoint M7

Highfield Campus

University of Southampton

Southampton, SO17 1BJ, UK

e-mail : O.Katsamenis@soton.ac.uk

\section{Keywords}

$X$-ray microfocus Computed Tomography $(\mu C T)$, 3D printing (3DP), structural characterisation, functional characterisation, Non-Destructive Testing (NDT)

\begin{abstract}
One of the most promising advances in modern pharmaceutical technology is the introduction of three-dimensional (3D) printing technology for the fabrication of drug products. 3D printed dosage forms have the potential to revolutionize pharmacotherapy as streamlined production of structurally complex formulations with optimal drug releasing properties is now made possible. 3D printed
\end{abstract}

This article has been accepted for publication and undergone full peer review but has not been through the copyediting, typesetting, pagination and proofreading process, which may lead to differences between this version and the Version of Record. Please cite this article as doi: 10.1111/jmi.12798.

This article is protected by copyright. All rights reserved. 
formulations are derived as part of a process where a "print-head" deposits, or sinters material under computer control to produce a drug carrier. However, this manufacturing route inherently generates objects that deviate from the ideal designed template for reasons specific to the 3D printing method used. This short opinion article discusses the potential of high-resolution nondestructive 3D (volume) imaging by means of $X$-ray microfocus Computed Tomography $(\mu C T)$ as a Process Analytical Technology for the structural and functional characterisation of 3D printed dosage forms.

\section{Introduction}

One of the most promising advances in modern pharmaceutical technology is the introduction of 3D printing (3DP) for the fabrication of formulations that have the potential to revolutionize pharmacotherapy, making possible both personalization of medication and efficient production of dosage forms with sophisticated designs (Gioumouxouzis et al., 2019). The 2015 approval from the United States Food and Drug Administration (FDA) of the first 3D printed drug product, signified the official opening of this new era (Norman et al., 2017). 3D printing technology can significantly improve healthcare at three distinct domains: 1 - at the community pharmacy domain, fabrication of polypills for patients receiving a range of medications daily can significantly improve patient compliance and comfort. 2 - at hospital pharmacy, on-site production by means of 3DP can enable pharmacy departments to personalize medication, by printing dosage forms containing the exact amount of Active Pharmaceutical Ingredient (API) tailored to each patient's need; an approach particularly beneficial for APIs with low therapeutic index. 3 - at an industrial level where streamlined production of structurally complex formulations, previously impractical to produce in a cost-effective manner, can now be made possible, along with the production of standardized APIloaded feedstock materials, like filament spools or bioink cartridges for the aforementioned categories. A combination of any of the above benefits could apply at any of the above domains, depending upon the prescribed medications and the capabilities of the producing facility.

\section{Sources of dimensional variability of 3D printed dosage forms}

Being a new manufacturing technique in the field of pharmaceutical technology, 3D printing needs to undergo extensive validation before it can be widely applied in routine production of dosage forms. The nature of this preparation method is substantially different compared to other manufacturing methods, like direct compression, in which dosage forms are formulated in moulds with well-defined shape and dimensions during a rapid process.

3D printed formulations are derived as part of a process where a print-head is [a] depositing molten polymer-based material (Fused Deposition Modeling-FDM) (Gioumouxouzis et al., 2018a) or semisolid pastes/bioinks (Extrusion/Inkjet Printing-EP/IP) (Kyobula et al., 2017), or [b] sinters powder using a laser beam (Selective Laser Sintering-SLS) (Fina et al., 2017), or a binding liquid (Powder Bed Printing-PBP) (Yu et al., 2007), or [c] solidifies liquid resins via UV emission (Stereolithography-SLA) (Wang et al., 2016). These processes inherently create objects with deviations from the ideal designed template for reasons specific to each 3D printing method. In FDM deviations can occur as a result of the behaviour of the printed material when is still in a semi-solid form. For example: 
Thermal or mechanical deformation of the printed polymeric building-block strands during molten polymer deposition from the printhead can significantly alter their shape. Deformations can be present in extrusion or inkjet 3DP especially when the printed material does not possess the ideal viscosity or solidifies slowly. Fusion between layers can be suboptimal, resulting in unwanted internal pores, while variability in feedstock material rheology can lead to inconsistent extrusion patterns and material agglomerations. All these can have a compounding effect on the geometrical inaccuracy of the printed object (Gioumouxouzis et al., 2018a, Gioumouxouzis et al., 2018b).

Increased surface roughness, attributed to the succession of layers (a phenomenon that diminishes as printer resolution increases), is another source of deviation from the designed object template. In SLS or PBP printing surface roughness and internal porosity are inherent characteristics, as prints consist essentially of powder aggregates sintered by a laser beam or bonded by a sprayed liquid, respectively.

Drug-loaded filaments used as feedstock material in FDM 3DP are produced via Hot-melt Extrusion (HME) and such processing may result in air bubble entrapment in the filament (internal porosity). Improper flow of the molten polymer from the HME nozzle (mainly due to high local stress causing a stress singularity at the die exit) can also result in substantial filament surface roughness ("sharkskin" effect) (Miller \& Rothstein, 2004).

\section{Characterisation by means of microfocus Computed Tomography}

Process analytical technology or "PAT" is defined by FDA and describes a regulatory framework that encourages the development and implementation of pharmaceutical development, manufacturing, and quality assurance methods (Food and Drug Administration, 2004). On this basis a number of techniques including thermal imaging, spectroscopy, interferometry, and ultrasonic inspection have been applied as PAT for 3D printing (Norman et al., 2017). However, none of these methods are able to provide high-resolution volumetric characterisation of the 3DP dosage forms.

High resolution non-destructive 3D (volume) imaging can be employed to verify that formulations comply with the required specifications (i.e. their dimensions are within tolerance from their templates), to access porosity and evaluate presence or absence of internal defects. One such prominent method of characterisation is X-ray microfocus Computed Tomography or micro-CT $(\mu \mathrm{CT})$. $\mu C T$, similarly to the clinical $C T$, measures the attenuation of $X$-rays through an object around an axis of rotation. Imaging is accomplished by placing a sample in the path of an X-ray beam and capturing its projection image (radiograph) over a large number of equidistant angles (typically in the order of thousands depending on detector and sample characteristics). Upon completion, the set of radiographs is fed into reconstruction algorithms, which use this information to calculate the x-ray attenuation at each point (elemental sub-volume) within the scanned volume. The calculated attenuation coefficient, is affected by the number of photons scattered or absorbed into these subvolumes as they pass through the object, and is depended upon the electron density of the tissue within this sub-volume. The calculated value per elemental sub-volume is then expressed as voxel (3D pixel) intensity in the reconstructed volume image domain, so that brighter voxels indicate areas of higher attenuation coefficient than darker ones. The result is a high-resolution volume image, composed of consequent 2D trans-axial images of the object (Landis \& Keane, 2010, Kalender, 2011).

This article is protected by copyright. All rights reserved. 
This enables visualization of both the external and the internal structure of the objects with exceptional resolution; typical voxel-size in the order of a few micrometres, although for much smaller volumes of interest voxel-size can be $<1 \mu \mathrm{m}$. The technique can perform detailed mapping of the external surface of 3D printed dosage forms and assess their porosity, both qualitatively (Sadia et al., 2018, Arafat et al., 2018, Goyanes et al., 2018, Smith et al., 2018, Alhijjaj et al., 2016, Holländer et al., 2018) and quantitatively (Markl et al., 2017, Gioumouxouzis et al., 2018a, Verstraete et al., 2018, Fina et al., 2018a, Fina et al., 2018b, Fina et al., 2017). Such assessment can include pore distribution, pore morphology and size, calculation of total porosity, or evaluation of surface- and sub-surface pores and pore interconnectivity.

Shape variations due to imperfect printing for example can be quantified by means of nominal (Computer-aided design, CAD) vs actual (printed object) comparison, where the $\mu \mathrm{CT}$ imaging data of the printed object are co-registered with the CAD data used to for the printing and compared by applying logical operators. An example of such operators is described by Markl et al. and presented in Figure 1 (Markl et al., 2017). The workflow allows for the identification of a sub-volume within either the $\mu \mathrm{CT}$ which does not overlap with the CAD model (XOR-X $\mu \mathrm{CT}$ data) and vice versa (XORCAD data). In simple terms exclusive or (XOR) data represent the overall variation between the design and the print, $\mathrm{XOR}-\mathrm{X} \mu \mathrm{CT}$ represents printed structures that were not meant to be printed (e.g. excess material) and XOR-CAD data the areas where material should have been present but it is not (e.g. pores).

An intriguing capability of $\mu \mathrm{CT}$ is its ability for not only morphological and dimensional characterisation, but also functional characterisation of the printed dosage forms, via time-resolved imaging - also referred to as 4D-CT. Function-specific experiments (e.g. dissolution, hydration, etc) can be performed in situ or ex situ during $\mu \mathrm{CT}$ imaging, providing valuable insights of the behaviour of the structure and its constituent component over-time (Gioumouxouzis et al., 2018b,

Gioumouxouzis et al., 2017). Such an approach is beneficial for accessing the dissolution behaviour of the 3D printed dosage forms. $\mu \mathrm{CT}$ can visualise disintegration and wetting of the formulation, as well as internal and external pore merging or collapse over-time.

In the research domain, being non-destructive, $\mu \mathrm{CT}$ allows for dosage forms to be imaged and consequently to be returned to the laboratory for further analysis with complementary techniques such as Scanning Electron Microscopy (SEM), or mechanical testing. Importantly, the same also applies to the characterisation of the drug-loaded filaments used as feedstock material in FDM 3DP, where $\mu \mathrm{CT}$ can be a used to ensure filament's homogeneity and dimensional consistency (Smith et al., 2018).

On the production line in the near future, combination of rapid $\mu \mathrm{CT}$ imaging and computer vision could be used on-the-fly for quality control of large dosage forms batches or bespoke personalized drug-delivery systems.

\section{Conclusion}

It is the authors belief that 3DP pharmaceutical formulations are expected to enter dynamically the market of personalized pharmacotherapy in the forthcoming decade. 3D printing has the potential to revolutionize pharmacotherapy, not only by personalizing the administration and dosing of everyday medication, but also by reducing shipping and distribution costs and by creating dosage

This article is protected by copyright. All rights reserved. 
forms with an unprecedented variability and complexity, previously impossible to create in a costand time-effective manner. In that direction, regulatory authorities should establish guidelines and specifications regarding quality assurance of 3D printed formulation (e.g., deviation tolerance of the printed formulations from their templates, accepted friability, hardness, etc) and rigorous evaluating procedures able to provide precise characterisation of the 3DP formulation should be employed at both the research and the industrial domain.

So far utilization of $\mu \mathrm{CT}$ in 3DP applications for pharmaceutical technology has primarily focused on qualitative inspection of the produced dosage forms and in some cases evaluation of porosity. While these aspects are highly valuable, they barely skim the surface of the potential of this imaging technology. $\mu \mathrm{CT}$ is a powerful tool that also allows for quantitative 3D (volume) imaging of the dosage forms' structure for dimensional metrology, but most importantly, because of its nondestructive nature it offers a unique window into the functional performance of the formulation over time. The latter can be used to gain valuable insight into the structural changes that take place during a certain process (e.g. dissolution, hydration, disintegration) and is of utmost importance, as 3DP printed formulations are often designed with complex internal structure which is critical for their release properties.

$\mu \mathrm{CT}$ is a powerful tool for standardized characterisation of 3D printed pharmaceuticals. We strongly believe that $\mu \mathrm{CT}$ can revolutionize the way structural and functional characterisation of 3DP formulations is performed and play a significant role in acceleration of early adoption of 3DP technology by the wider pharmaceutical technology sector.

\section{Footnote}

This opinion article is related to our paper presented at the $6^{\text {th }}$ annual Tomography for Scientific Advancement (ToScA) where we were honoured with the "best lightning talk" award. The presented paper (poster) can be downloaded from the following link:

https://cdn.southampton.ac.uk/assets/imported/transforms/contentblock/UsefulDownloads Download/E673695E1BC4423CBACAD82F9C1EFA28/Katsamenis et al ToS cA 2018.pdf\# ga=2.251969613.572331451.1542570158-1103405630.1394233722

\section{Acknowledgements}

We would like to acknowledge $\mu$-VIS X-Ray Imaging Centre at the University of Southampton for the provision of tomographic imaging facilities, as well as Nikon X-Tek Systems Ltd for the provision of the Med-X prototype scanner used in (Gioumouxouzis et al., 2018b).

This article is protected by copyright. All rights reserved. 


\section{References}

Alhijjaj, M., Belton, P. \& Qi, S. (2016) An investigation into the use of polymer blends to improve the printability of and regulate drug release from pharmaceutical solid dispersions prepared via fused deposition modeling (FDM) 3D printing. European Journal of Pharmaceutics and Biopharmaceutics, 108, 111-125.

Arafat, B., Wojsz, M., Isreb, A., Forbes, R. T., Isreb, M., Ahmed, W., Arafat, T. \& Alhnan, M. A. (2018) Tablet fragmentation without a disintegrant: A novel design approach for accelerating disintegration and drug release from 3D printed cellulosic tablets. European Journal of Pharmaceutical Sciences, 118, 191-199.

Fina, F., Goyanes, A., Gaisford, S. \& Basit, A. W. (2017) Selective laser sintering (SLS) 3D printing of medicines. International journal of pharmaceutics, 529, 285-293.

Fina, F., Goyanes, A., Madla, C. M., Awad, A., Trenfield, S. J., Kuek, J. M., Patel, P., Gaisford, S. \& Basit, A. W. (2018a) 3D printing of drug-loaded gyroid lattices using selective laser sintering. International journal of pharmaceutics.

Fina, F., Madla, C. M., Goyanes, A., Zhang, J., Gaisford, S. \& Basit, A. W. (2018b) Fabricating 3D printed orally disintegrating printlets using selective laser sintering. International journal of pharmaceutics, 541, 101-107.

Food and Drug Administration, F. (2004) Guidance for industry, PAT-A Framework for Innovative Pharmaceutical Development, Manufacturing and Quality Assurance. https://www.fda.gov/downloads/Drugs/GuidanceComplianceRegulatory/nformation/Guidan ces/ucm070305.pdf.

Gioumouxouzis, C. I., Baklavaridis, A., Katsamenis, O. L., Markopoulou, C. K., Bouropoulos, N., Tzetzis, D. \& Fatouros, D. G. (2018a) A 3D printed bilayer oral solid dosage form combining metformin for prolonged and glimepiride for immediate drug delivery. European Journal of Pharmaceutical Sciences, 120, 40-52.

Gioumouxouzis, C. I., Chatzitaki, A.-T., Karavasili, C., Katsamenis, O. L., Tzetzis, D., Mystiridou, E., Bouropoulos, N. \& Fatouros, D. G. (2018b) Controlled Release of 5-Fluorouracil from Alginate Beads Encapsulated in 3D Printed pH-Responsive Solid Dosage Forms. AAPS PharmSciTech, 1-14.

Gioumouxouzis, C. I., Karavasili, C. \& Fatouros, D. G. (2019) Recent advances in pharmaceutical dosage forms and devices using additive manufacturing technologies. Drug Discov Today, 24, 636-643.

Gioumouxouzis, C. I., Katsamenis, O. L., Bouropoulos, N. \& Fatouros, D. G. (2017) 3D printed oral solid dosage forms containing hydrochlorothiazide for controlled drug delivery. Journal of Drug Delivery Science and Technology, 40, 164-171.

Goyanes, A., Fernández-Ferreiro, A., Majeed, A., Gomez-Lado, N., Awad, A., Luaces-Rodríguez, A., Gaisford, S., Aguiar, P. \& Basit, A. W. (2018) PET/CT imaging of 3D printed devices in the gastrointestinal tract of rodents. International journal of pharmaceutics, 536, 158-164.

Holländer, J., Hakala, R., Suominen, J., Moritz, N., Yliruusi, J. \& Sandler, N. (2018) 3D printed UV light cured polydimethylsiloxane devices for drug delivery. International journal of pharmaceutics, 544, 433-442.

Kalender, W. A. (2011) Computed tomography: fundamentals, system technology, image quality, applications, John Wiley \& Sons.

Kyobula, M., Adedeji, A., Alexander, M. R., Saleh, E., Wildman, R., Ashcroft, I., Gellert, P. R. \& Roberts, C. J. (2017) 3D inkjet printing of tablets exploiting bespoke complex geometries for controlled and tuneable drug release. Journal of Controlled Release, 261, 207-215.

Landis, E. N. \& Keane, D. T. (2010) X-ray microtomography. Materials characterization, 61, 13051316.

Markl, D., Zeitler, J. A., Rasch, C., Michaelsen, M. H., Müllertz, A., Rantanen, J., Rades, T. \& Bøtker, J. (2017) Analysis of 3D prints by x-ray computed microtomography and terahertz pulsed imaging. Pharmaceutical research, 34, 1037-1052.

This article is protected by copyright. All rights reserved. 
Miller, E. \& Rothstein, J. P. (2004) Control of the sharkskin instability in the extrusion of polymer melts using induced temperature gradients. Rheologica acta, 44, 160-173.

Norman, J., Madurawe, R. D., Moore, C. M. V., Khan, M. A. \& Khairuzzaman, A. (2017) A new chapter in pharmaceutical manufacturing: 3D-printed drug products. Advanced drug delivery reviews, 108, 39-50.

Sadia, M., Arafat, B., Ahmed, W., Forbes, R. T. \& Alhnan, M. A. (2018) Channelled tablets: An innovative approach to accelerating drug release from 3D printed tablets. Journal of Controlled Release, 269, 355-363.

Smith, D. M., Kapoor, Y., Klinzing, G. R. \& Procopio, A. T. (2018) Pharmaceutical 3D printing: Design and qualification of a single step print and fill capsule. International journal of pharmaceutics, 544, 21-30.

Verstraete, G., Samaro, A., Grymonpré, W., Vanhoorne, V., Van Snick, B., Boone, M. N., Hellemans, T., Van Hoorebeke, L., Remon, J. P. \& Vervaet, C. (2018) 3D printing of high drug loaded dosage forms using thermoplastic polyurethanes. International journal of pharmaceutics, 536, 318-325.

Wang, J., Goyanes, A., Gaisford, S. \& Basit, A. W. (2016) Stereolithographic (SLA) 3D printing of oral modified-release dosage forms. Int J Pharm, 503, 207-212.

Yu, D. G., Yang, X. L., Huang, W. D., Liu, J., Wang, Y. G. \& Xu, H. (2007) Tablets with material gradients fabricated by three-dimensional printing. J Pharm Sci, 96, 2446-2456.

\section{Figure Legends}

Figure 1: Schematic of the example logical operators used for nominal (CAD) vs actual (printed object) comparison. The chart describes a workflow that allows for quantitative assessment of the deviation of the printed object from the CAD design. Circles are used to denote the volume of the "real" (i.e. printed) and "designed" (i.e. CAD) object and rectangles to denote logical operations between the aforementioned sets of data the results of which quantify the printing accuracy. Adapted from reference (Markl et al., 2017).

Figure 2: Representative examples of quantitative dimensional metrology analysis (Case study 1) and functional characterisation by means of time-resolved imaging (Case study 2) of 3DP dosage forms. Case Study 1: [a-d] Deviation map of printed object's surface from the nominal values of the CAD design; [e-g] CT slices through the PVA layer, the PVA/ Eudragit ${ }^{\circledR}$ layer interface and the centre of Eudragit ${ }^{\circledR} ;$ [h] cross-section through the XZ plane Sample: flat cylindrical with smoothed edges; Constituents: water-soluble PVA (+ glimepiride), Eudragit ${ }^{\circledR}$ RL (+ Metformin); Case Study 2: top row: Sum along the Rays renderings of the printed form at its initial/dry state (scan 1), after $2 \mathrm{~h}$ exposure to simulated gastric fluid, SGF (scan 2), consequent $2 \mathrm{~h}$ exposure to simulated intestinal fluid, SIF (scan 3), followed by $5 \mathrm{~h}$ exposure to phosphate buffer saline, PBS (scan 4). Bottom row: CT slices through the Eudragit ${ }^{\circledR}$-based layer before and after the $9 \mathrm{~h}$ exposure to the various solutions ; Sample: Eudragit ${ }^{\circledR}$ L100-55 / Eudragit ${ }^{\circledR}$ S100 (pH responsive layer), polylactic acid (PLA), alginate particles (+5-Fluorouracil); Adapted with permission from references (Gioumouxouzis et al., 2018a, Gioumouxouzis et al., 2018b).

This article is protected by copyright. All rights reserved. 
Figures

Figure 1
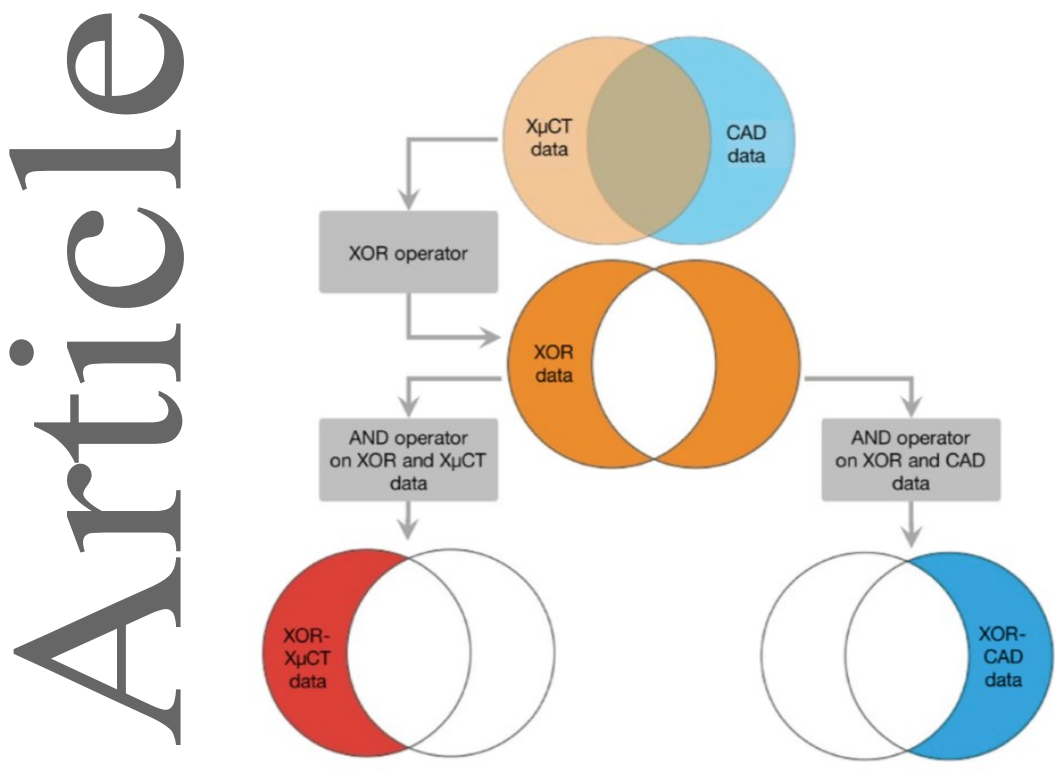

Figure 2

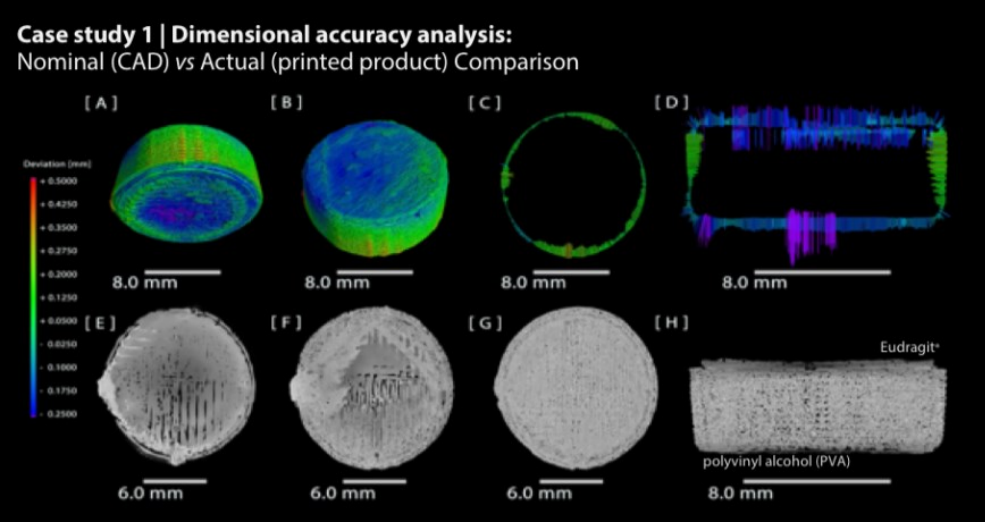

Case study 2 | Functional characterisation:

Controlled drug release from alginate beads encapsulated in $\mathrm{pH}$-responsive 3D printed carrier

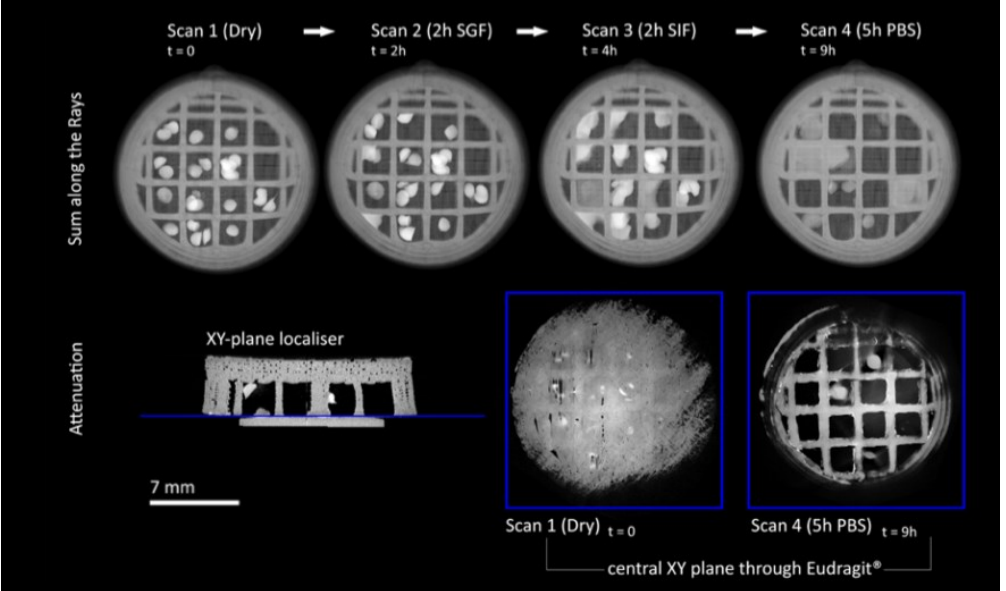

This article is protected by copyright. All rights reserved. 